\title{
Downregulation of Meg3 enhances cisplatin resistance of lung cancer cells through activation of the WNT/ $\beta$-catenin signaling pathway
}

\author{
YANG XIA*, ZHICHENG HE* , BIN LIU, PENGLI WANG and YIJIANG CHEN \\ Department of Thoracic and Cardiovascular Surgery, The First Affiliated Hospital of Nanjing Medical University, \\ Nanjing, Jiangsu 210000, P.R. China
}

Received June 14, 2014; Accepted March 3, 2015

DOI: $10.3892 / \mathrm{mmr} .2015 .3897$

\begin{abstract}
Maternally expressed gene 3 (Meg3) has been shown to promote tumor progression. However, the role of Meg3 in the development of a chemoresistant phenotype of human lung cancer has remains. Reverse transcription-quantitative polymerase chain reaction analysis was used to determine the expression of Meg3. Flow cytometric analysis and MTT assay were also used to investigate the cell cycle and apoptosis. The present study detected that the expression levels of Meg3 were significantly lower in cisplatin-resistant A549/DDP lung cancer cells, compared with those in parental A549 cells. Furthermore, upregulation of Meg3 was able to re-sensitize the A549/DDP cells to cisplatin in vitro. Whereas downregulation of Meg3, by RNA interference, decreased the sensitivity of A549 cells to cisplatin. The results of the present study also demonstrated that the Meg3-mediated chemosensitivity enhancement was associated with the induction of cell-cycle arrest and increased apoptosis, through regulation of $\mathrm{p} 53, \beta$-catenin and survivin, which is a target gene of the WNT/ $\beta$-catenin signaling pathway. In conclusion, these results suggested that Meg3 may have a crucial role in the development of cisplatin resistance in non-small cell lung cancer.
\end{abstract}

\section{Introduction}

Lung cancer is one of the most common causes of cancer-associated mortality worldwide (1). Non-small cell lung cancer (NSCLC) is predominantly divided into squamous cell

Correspondence to: Dr Yijiang Chen, Department of Thoracic and Cardiovascular Surgery, The First Affiliated Hospital of Nanjing Medical University, 300 Guangzhou Road, Nanjing, Jiangsu 210000, P.R. China

E-mail: yjchen@njmu.edu.cn

${ }^{*}$ Contributed equally

Key words: maternally expressed gene 3, apoptosis, cell cycle, cisplatin, A549/DDP, lung cancer carcinoma and adenocarcinoma (1). The five-year survival rate for NSCLC is $\sim 10-15 \%$ (2). The postoperative resistance of patients with lung cancer to chemotherapy drugs, including cisplatin and tyrosine kinase inhibitors (TKI), is a significant factor influencing disease prognosis. Therefore, exploration into chemotherapy resistance may help improve the treatment of patients with lung cancer.

Long non-coding RNAs (lncRNAs) belong to a novel heterogeneous class of ncRNAs, and are involved in various biological processes, including imprinting (3), histone-code regulation and proliferation (4) of cancer cells, through regulation of gene expression (5). IncRNA H19 is able to regulate the induction of multidrug resistance protein 1-associated drug resistance in hepatocellular carcinoma cells (6). A previous study demonstrated that lncRNA UCA1 may enhance the chemoresistance of bladder cancer cells via regulation of WNT signaling (7). A novel lncRNA ARA has been shown to contribute to adriamycin resistance in cancer, through the modulation of numerous signaling pathways (8). Maternally expressed gene 3 (Meg3) is a tumor suppressor gene that encodes an lncRNA and is expressed in numerous types of normal tissue; however, its expression is lost in multiple cancer cell lines that originate from various types of tissue (9). Previous studies have demonstrated that Meg3 may inhibit proliferation (10-12) and induce apoptosis $(10,13)$ of tumor cells in vitro. However, little is currently known regarding the role of Meg3 in the development of chemoresistant phenotypes of lung cancer. Cisplatin is a commonly used anti-cancer drug, which induces apoptosis by suppressing the DNA replication process of cancer cells (14).

Alterations in the $\mathrm{WNT} / \beta$-catenin signaling pathway are frequent amongst human malignancies. In NSCLC, mutations in $\beta$-catenin are uncommon; however, WNT signaling is significant in NSCLC cell lines and WNT inhibition results in increased levels of apoptosis (15). WNT signaling has a substantial impact on NSCLC tumorigenesis, prognosis and resistance to therapy, and a loss of WNT signaling inhibitors by promoter hypermethylation or other mechanisms appears to be particularly important (15).

It was hypothesized that Meg3 may regulate apoptosis, cell cycle distribution and drug resistance of cancer cells via the inhibition of $\beta$-catenin/survivin by activated $\mathrm{p} 53$. The present 
study therefore aimed to elucidate the mechanisms underlying the effects of Meg3 on apoptosis, cell cycle and drug resistance in NSCLC. The expression levels of Meg3 in A549/DDP cisplatin-resistant lung cancer cells and A549 parental lung cancer cells were evaluated and; furthermore, Meg3-mediated chemosensitivity enhancement was investigated in these cell lines.

\section{Materials and methods}

Cell culture and blood samples. A549 and A549/DDP human lung cancer cell lines were used in the present study. The cells were cultured in RPMI-1640 medium (Invitrogen Life Technologies, Carlsbad, CA, USA) supplemented with $10 \%$ fetal bovine serum (FBS; Invitrogen Life Technologies) and penicillin $\left(100 \mathrm{U} / \mathrm{ml}\right.$; Invitrogen Life Technologies), at $37^{\circ} \mathrm{C}$ in an atmosphere containing $5 \% \mathrm{CO}_{2}$. Blood samples $(\mathrm{n}=27)$ were collected from patients with advanced lung cancer that had received cisplatin-based chemotherapy between May 2010 and December 2012, at the Affiliated Hospital of Jiangnan University (Wuxi, China). The median patient age was 64 (range, 49-85 years). The patients included 16 females and 11 males. All specimens were histologically classified by a professional pathologist, according to the national NCCN guidelines for NSCLC version 3.2011 (16) in a blinded manner. Written informed consent was obtained from all of the patients recruited. The methodologies of the present study conformed to the standards set by the Declaration of Helsinki; and the study was approved by the Ethical Committee of the Affiliated Hospital of Jiangnan University (Wuxi, China)

Plasmid construction and cell transfection. The sequence of Meg3 was synthesized by Genewiz (Suzhou, China). All recombinant lentiviruses were produced by calcium phosphate-mediated transient transfection of HEK293T cells, according to standard protocols. Briefly, HEK293T cells from Clontech were cultured in Dulbecco's modified Eagle's medium (DMEM), supplemented with 10\% FBS and penicillin/streptomycin $(100 \mathrm{U} / \mathrm{ml})$. The subconfluent cells in a $10 \mathrm{~cm}$ culture dish were co-transfected with lentiviral vector $(10 \mu \mathrm{g})$, the lentiviral packaging vectors pLv-GFP or pLv-Meg3 $(2 \mu \mathrm{g}), \mathrm{pMDLg} / \mathrm{pRRE}(5 \mu \mathrm{g})$, and the vesicular stomatitis virus $\mathrm{G}$ glycoprotein (VSVG) expression vector pMD2G $(3 \mu \mathrm{g})$. The viruses were collected from the culture supernatants on days 2 and 3 post-transfection. The cultured cells were incubated with lentivirus at various concentrations for $24 \mathrm{~h}$ and cultured with DMEM, containing 10\% FBS. A pure population of infected cells were sorted based on the GFP-expression by flow cytometry and $>98 \%$ of the cells were GFP-positive following sorting. The plasmid was subcloned into a lentiviral vector, and then co-transfected into HEK-293T human embryonic kidney cells using the Lentiviral Packaging Mix. A549/DDP cells were transfected with the lentivirus, and cells which highly expressed Meg3 (A549/DDP-Meg3 cells) were identified by selection with G418. The Meg3 sequence (NR_002766.2) was subcloned into an HIV type-1 lentiviral vector, pLV-GFP (Addgene, Cambridge, MA, USA) by restriction digestion using BamHI and MluI (New England Biolabs, Ltd., Hertfordshire, United Kingdom). The resulting constructs was termed pLV-Meg3 and pLV-GFP was used as a control. Following trypsinization, the cells were centrifuged at $1000 \mathrm{x} g$ for $5 \mathrm{~min}$ and the supernatant was removed. A total of $1 \times 10^{6}$ cells were subsequently seeded into six-well plates at high density (80-90\% confluent). Lipofectamine 2000 (Invitrogen Life Technologies) complexes were prepared, according to the standard Lipofectamine protocol. Lipofectamine 2000 reagent $(10 \mu \mathrm{l})$ and 100 pmol siRNA were each diluted in 250 $\mu$ l Opti-MEM medium. Each mix was pooled and incubated for 5 min prior to the DNA-reagent complex being added to the cells (500 $\mu \mathrm{l}$ per well). The cells were incubated for $6 \mathrm{~h}$ at $37^{\circ} \mathrm{C}$ and subsequently incubated with DMEM, containing $10 \%$ FBS for $24 \mathrm{~h}$.

Transfection of small interfering (si)RNAs. The A549 cells were seeded at 50-80\% cell density into six-well plates and transfected with $50 \mathrm{nM}$ siRNAs specifically targeting Meg3 (siRNA/Meg3-243: 5'-GAUCCCACCAACAUACAAATT-3'; siRNA/Meg3-405: 5'-GCUCAUACUUUGACUCUAUTT-3'; siRNA/Meg3-852: 5'-CCCUCUUGCUUGUCUUACUTT-3') (Shanghai Gene Pharma Co., Ltd., Shanghai, China) using Lipofectamine ${ }^{\circledR} 2000$ (Invitrogen Life Technologies), according to the manufacturer's instructions, and were subsequently incubated for $24 \mathrm{~h}$ at $37^{\circ} \mathrm{C}$ in a $5 \% \mathrm{CO}_{2}$ incubator. The control siRNA sequence was as follows: 5'-UUAAGACGGUUGAAACUAG-3'.

Isolation of total RNA and reverse transcription-quantitative polymerase chain reaction $(R T-q P C R)$. Total RNA was extracted from the cells and blood samples using TRIzol ${ }^{\circledR}$ (Invitrogen Life Technologies) and was subsequently reverse transcribed into cDNA using a reverse transcriptase kit (Takara Bio, Inc., Otsu, Japan). The relative mRNA expression levels of Meg3 were examined by RT-qPCR using SYBR Premix Ex Taq (Takara Biotechnology Co., Ltd., Dalian, China), and normalized to GAPDH. The RT-qPCR was performed using the ABI 7500 Fast Real-Time PCR system (Applied Biosystems, Foster City, CA, USA). The PCR conditions were as follows: $94^{\circ} \mathrm{C}$ for $2 \mathrm{~min}, 94^{\circ} \mathrm{C}$ for $30 \mathrm{sec}, 60^{\circ} \mathrm{C}$ for $30 \mathrm{sec}$ and $72^{\circ} \mathrm{C}$ for $1 \mathrm{~min}$ for 30 cycles, and $72^{\circ} \mathrm{C}$ for $10 \mathrm{~min}$. The PCR primer sequences used were as follows: MEG3, forward: 5'-ATCATCCGTCCACCTCCTTGTCTTC-3' and reverse: 5'-GTATGAGCATAGCAAAGGTCAGGGC-3'; GAPDH, forward: 5'-AATGCCTCCTGCACCACCAAC-3' and reverse: 5'-AAGGCCATGCCAGTGAGCTTC-3'. Calculations of expression were made using the $2^{-\Delta \Delta \mathrm{CT}}$ method.

Flow cytometric analysis of apoptosis and cell cycle distribution. Apoptosis of the cultured A549 and A549/DDP cells was evaluated using Annexin V labeling. An Annexin V-Allophycocyanin-Labeled Apoptosis Detection kit (Abcam, Cambridge, UK) was used according to the manufacturer's instructions. Staining with annexin V/PI was performed using an Annexin V-APC Apoptosis detection kit (Invitrogen Life Technologies). Briefly, an aliquot containing $2 \times 10^{6}$ cells was resuspended in $0.5 \mathrm{ml}$ binding buffer, labelled with $1 \mu \mathrm{l}$ annexin V-APC plus $5 \mu \mathrm{l}$ PI and were incubated for 10 min in the dark. The samples were immediately analyzed. Annexin negative and PI negative cells were designated as live cells and annexin positive and PI negative as early apoptotic cells. Staining with PI was performed using cell cycle detec- 
tion kit (invitrogene, USA). Briefly, the cells were centrifuged at $500 \times \mathrm{g}$ for $10 \mathrm{~min}$ at room temperature and the supernatant was removed. An aliquot of $\sim 1 \times 10^{6}$ was incubated in ethyl alcohol at $-20^{\circ} \mathrm{C}$ for $24 \mathrm{~h}$ and was subsequently incubated in $50 \mu \mathrm{g} / \mathrm{ml}$ PI in the dark, at room temperature for $30 \mathrm{~min}$. The cells undergoing cell-cycle analysis were stained with propidium iodide, using the BD Cycletest Plus DNA Reagent kit (BD Biosciences, Shanghai, China), according to the manufacturer's instructions. The results were analyzed using a FACScan flow cytometer (BD Biosciences). The percentage of cells in each of the $\operatorname{subG}_{0} / G_{1}, G_{1}, S$ or $G_{2} / M$ phases were counted and compared. Each experiment was performed at least three times.

MTT assay. The chemosensitivity of the cisplatin-resistant A549/DPP and parental A549 cells to cisplatin was determined by MTT assay. Briefly, the cells were seeded into 96-well plates $\left(3.5 \times 10^{3}\right.$ cells/well) and treated with various concentrations of cisplatin $(0,1,5,8,10,15,18,20,22$ and $24 \mu \mathrm{g} / \mathrm{ml})$. At $0,24,48$, 72 and $96 \mathrm{~h}$ post-cisplatin application, cell survival was assessed using $0.5 \mathrm{mg} / \mathrm{ml}$ MTT (Sigma-Aldrich, St. Louis, MO, USA) solution. Following a $4 \mathrm{~h}$ incubation at $37^{\circ} \mathrm{C}$, the medium was replaced with $150 \mu \mathrm{l}$ dimethyl sulfoxide (Sigma-Aldrich) and vortexed for $10 \mathrm{~min}$. The absorbance of each well at $490 \mathrm{~nm}$ was measured using a microplate reader 550 (Bio-Rad, Richmond, CA, USA). Each experiment was performed at least three times.

Western blot analysis. The A549/DDP cells were washed in phosphate-buffered saline (PBS) and lysed in radioimmunoprecipitation assay lysis buffer supplemented with a protease inhibitor cocktail (Roche Diagnostics, Mannheim, Germany). The total protein was quantified using a Bicinchoninic Acid Protein Assay kit (Beyotime Institute of Biotechnology, Haimen, China). Equal amounts (30 $\mu \mathrm{g})$ of the whole cell lysates were separated by 5\% SDS-PAGE (Beyotime Institute of Biotechnology, Jiangsu, China) and transferred onto polyvinylidene difluoride membranes (Merck Millipore $\mathrm{GmbH}$, Eschborn, Germany). The blots were blocked in bovine serum albumin (5\% w/v in PBS+0.1\% Tween 20) for $1 \mathrm{~h}$ at room temperature and immunostained with the following antibodies at $4^{\circ} \mathrm{C}$ overnight: Mouse anti-human p53 (1:2,000; monoclonal, cat. no. ab26), mouse anti-human $\beta$-catenin (1:1,000; monoclonal, cat. no. ab6301), mouse anti-human survivin (1:500; monoclonal, cat. no. ab118046) and mouse anti-human GAPDH (1:5,000; monoclonal, cat. no. ab9484). These antibodies were purchased from Abcam (Cambridge, MA, USA). The membranes were washed four times with PBS, containing $0.1 \%$ Tween 20 . The secondary antibody, goat anti-mouse (1:50,000; polyclonal, cat. no. ab97040; Abcam), was added in PBS, containing $0.1 \%$ Tween 20 for $1 \mathrm{~h}$ at $37^{\circ} \mathrm{C}$. The membranes were then washed three times for 15 min with PBS, containing $0.1 \%$ Tween 20 (Sigma-Aldrich). The results were visualized using an enhanced chemiluminescent detection system (Pierce ECL Substrate Western Blot Detection system; Thermo Fisher Scientific, Waltham, MA, USA) and using a Molecular Imager ChemiDoc XRS system (Bio-Rad Laboratories, Inc., Hercules, CA, USA).

Statistical analysis. Statistical analysis was performed using STATA 11 (StataCorp LP, College Station, TX, USA), and
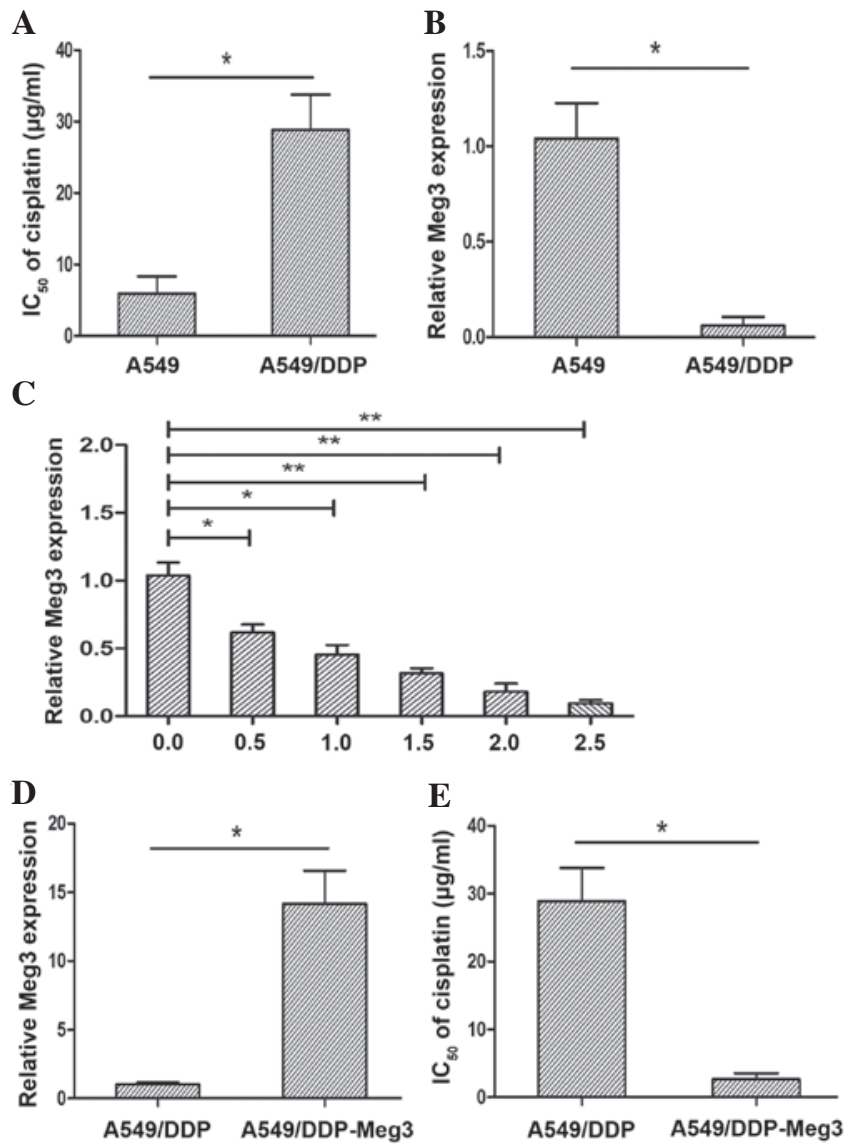

Figure 1. Expression levels of Meg3 were reduced in A549/DDP cisplatin-resistant human lung cancer cells. (A) $\mathrm{IC}_{50}$ value of cisplatin was higher in A549/DDP cells, as compared with that of the A549 cells. (B) Expression levels of Meg3 relative to GAPDH were determined in the A549/DDP and A549 cells by RT-qPCR. (C) A549 cells were cultured in various concentrations of cisplatin $(0.0,0.5,1.0,1.5,2.0$ or $2.5 \mu \mathrm{g} / \mathrm{ml})$ for $24 \mathrm{~h}$. Meg3 expression was evaluated by RT-qPCR. GAPDH was used as an internal control. (D) Meg3 expression was detected in A549/DDP cells stably transfected with a Meg3 lentivirus by RT-qPCR. GAPDH was used as an internal control. (E) $\mathrm{IC}_{50}$ values of cisplatin in A549/DDP and A549/DDP-Meg3 cells were analyzed by MTT assay. Data are presented as the mean \pm standard error of the mean. ${ }^{*} \mathrm{P}<0.05 ;{ }^{* *} \mathrm{P}<0.01$. $\mathrm{IC}_{50}$, half maximal inhibitory concentration; RT-qPCR, reverse transcription-quantitative polymerase chain reaction; Meg3, maternally expressed gene 3 .

presented using GraphPad Prism 4.0 software (GraphPad Software, Inc., La Jolla, CA, USA). The results obtained from the in vitro assays are presented as the mean \pm standard error of the mean from five separate experiments performed in triplicate. $\mathrm{P}<0.05$ was considered to indicate a statistically significant difference.

\section{Results}

Meg3 expression levels are markedly lower in the A549/DDP cisplatin-resistant cell line, compared with those in the A549 parental cell line. A549/DDP is a cisplatin-resistant lung cancer cell line. The present study conducted an MTT assay to determine the half maximal inhibitory concentration $\left(\mathrm{IC}_{50}\right)$ of cisplatin in the A549/DDP and parental A549 cell lines. The $\mathrm{IC}_{50}$ of cisplatin in the A549 and A549/CDDP cells was $5.68 \pm 2.54 \mu \mathrm{g} / \mathrm{ml}$ and $29.24 \pm 1.62 \mu \mathrm{g} / \mathrm{ml}$, respectively $(\mathrm{P}<0.05$; Fig. 1A). The A549/DDP cells exhibited a 5.15 -fold greater 
A
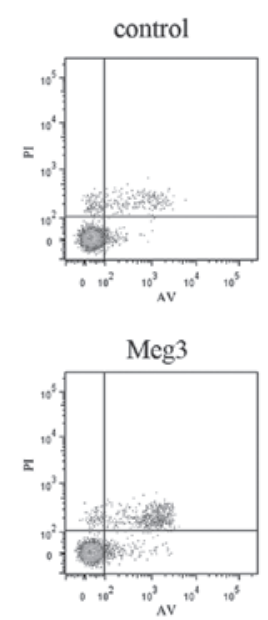

B

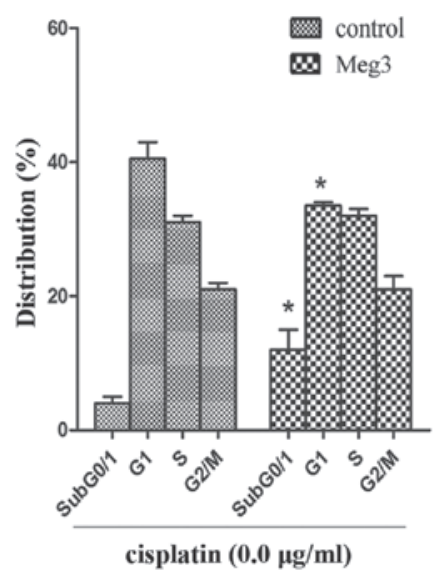

control+cisplatin

$(1.0 \mu \mathrm{g} / \mathrm{ml})$

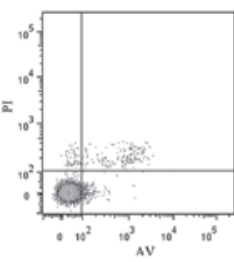

Meg3+cisplatin

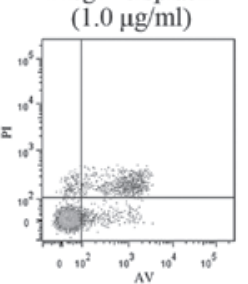

control+cisplatin

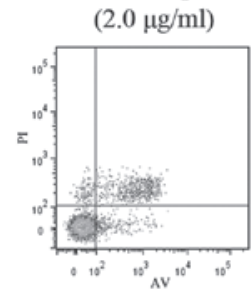

Meg3+cisplatin

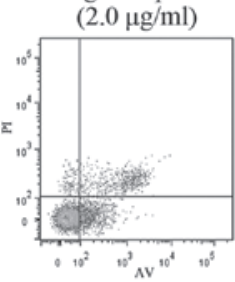

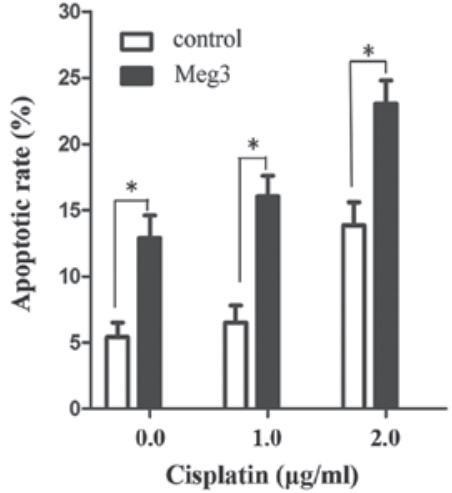

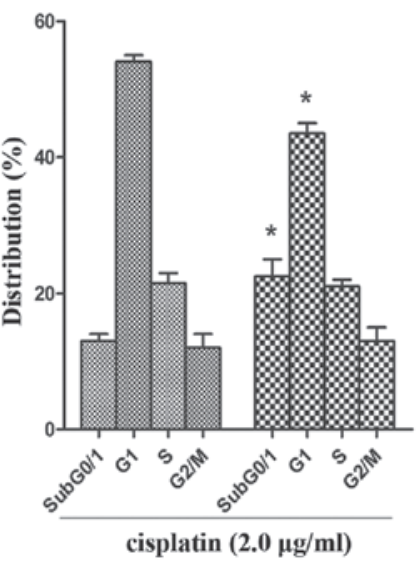

Figure 2. Meg3 increases the sensitivity of A549/DDP human lung cancer cells to cisplatin. (A) Flow cytometric analysis of apoptosis in A549/DDP (control) or A549/DDP-Meg3 (Meg3 overexpressing) cells treated with various concentrations of cisplatin (0.0, $1.0 \mathrm{or} 2.0 \mu \mathrm{g} / \mathrm{ml}$ ). (B) Flow cytometric analysis of the cell cycle distribution in A549/DDP or A549/DDP-Meg3 cells treated with various concentrations of cisplatin (0.0, 1.0 or $2.0 \mu \mathrm{g} / \mathrm{ml})$. The results represent the average of three independent experiments (mean \pm standard error of the mean). ${ }^{*} \mathrm{P}<0.05$, as compared with the control cells. Meg3, maternally expressed gene 3; PI, propidium iodide; AV, Annexin V.

A

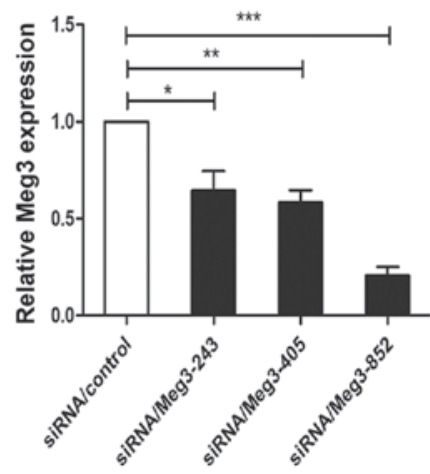

B

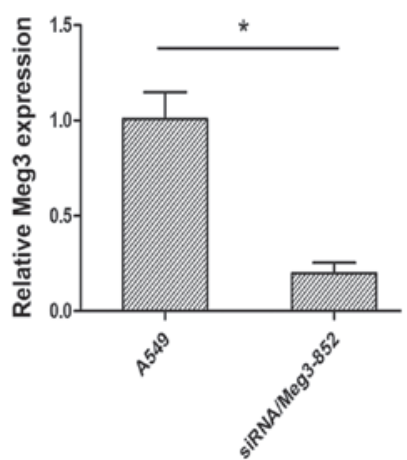

C

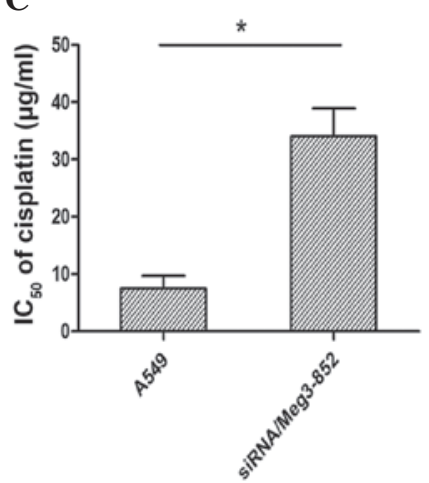

Figure 3. siRNA transfection reduces the expression of Meg3 and enhances the cisplatin-resistance of parental A549 cells. (A) A total of $48 \mathrm{~h}$ after the A549 human lung cancer cells were transfected with siRNA/control, siRNA/Meg3-243, siRNA/Meg3-405 or siRNA/Meg3-852, the expression levels of Meg3 were detected by RT-qPCR. GAPDH was used as an internal control. (B) Expression levels of Meg3 were detected to determine the inhibition efficiency of the siRNA by RT-qPCR in A549 and siRNA/Meg3-852 cells. (C) $\mathrm{IC}_{50}$ values of cisplatin in A549 and siRNA/Meg3-852 cells were analyzed using an MTT assay. Data are presented as the mean \pm standard error of the mean. ${ }^{*} \mathrm{P}<0.05,{ }^{* * *} \mathrm{P}<0.01,{ }^{* *} \mathrm{P}<0.001$. siRNA, small interfering RNA; RT-qPCR, reverse transcription-quantitative polymerase chain reaction; $\mathrm{IC}_{50}$, half maximal inhibitory concentration; Meg3, maternally expressed gene 3.

resistance to cisplatin compared with that of the parental A549 cell line. To further investigate whether Meg3 had a significant role in the acquired cisplatin resistance of lung cancer cells, the expression levels of Meg3 were detected in the A549/DDP and parental A549 cells by RT-qPCR. The expression levels of Meg3 were significantly lower in the A549/DDP cisplatin-resistant cell 
A
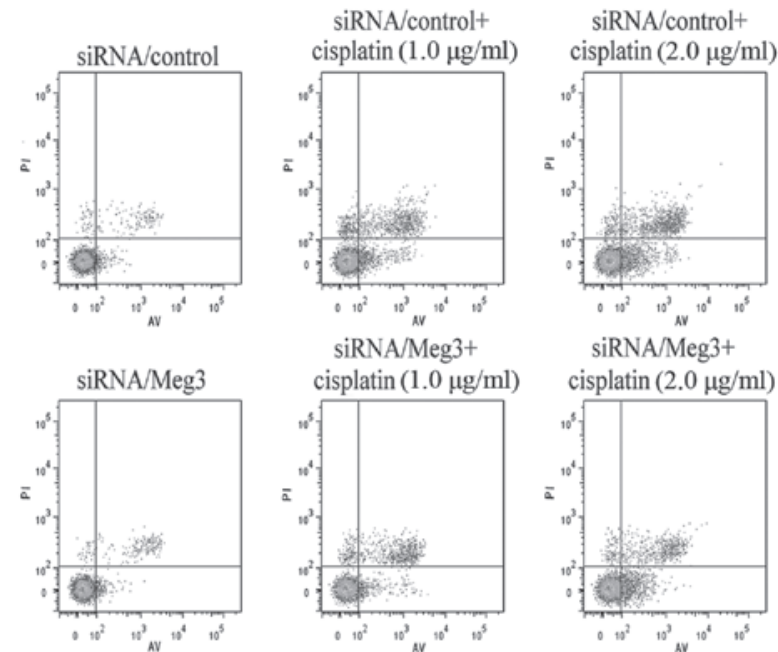

siRNA/Meg3+ cisplatin $(1.0 \mu \mathrm{g} / \mathrm{ml})$ siRNA/Meg3+ cisplatin $(2.0 \mu \mathrm{g} / \mathrm{ml})$

B
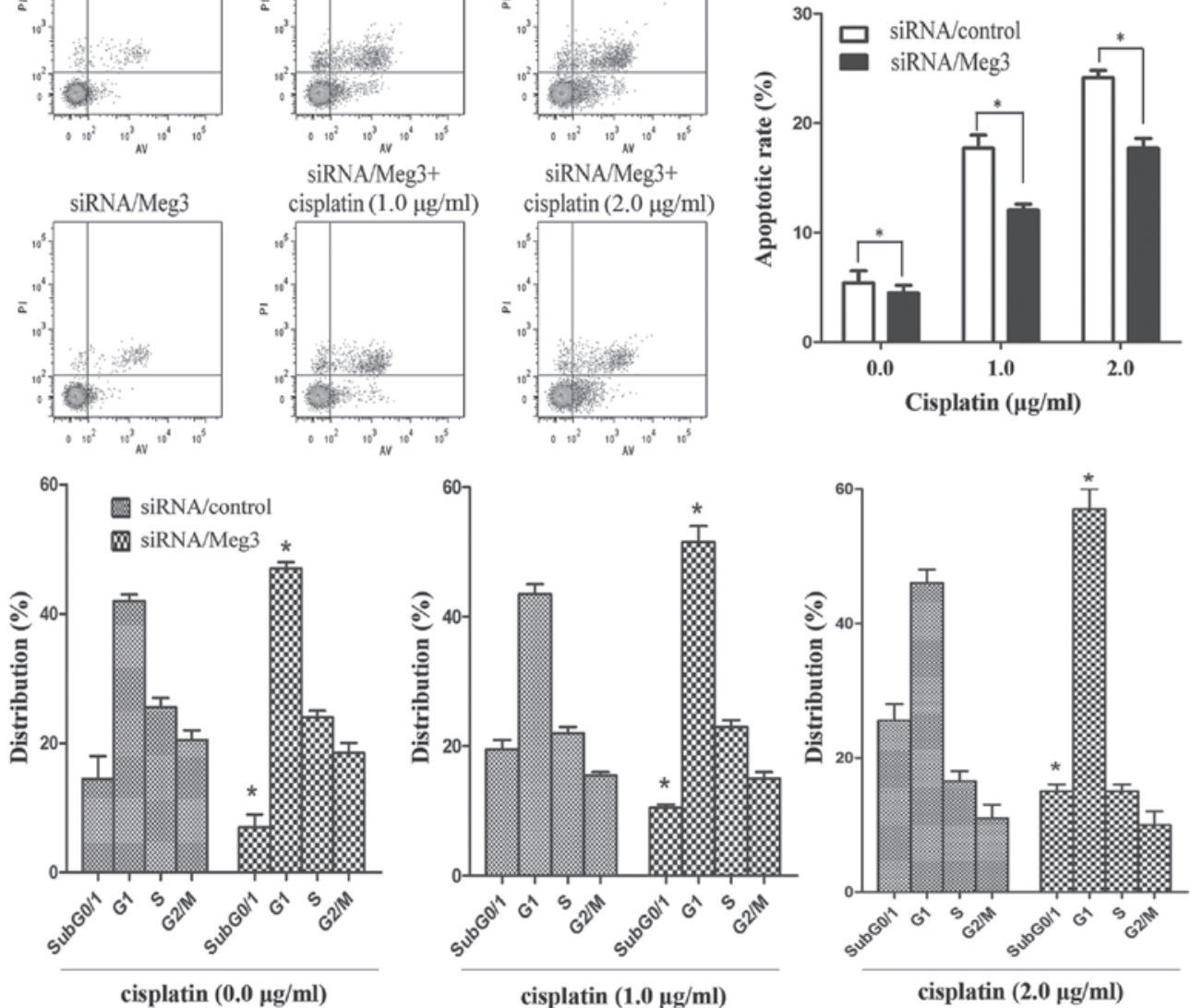

cisplatin $(1.0 \mu \mathrm{g} / \mathrm{ml})$

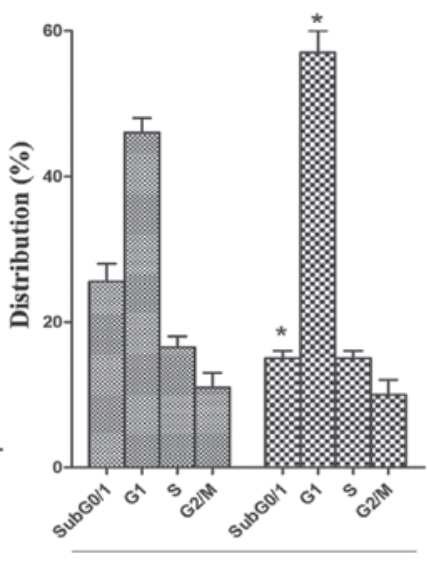

cisplatin $(2.0 \mu \mathrm{g} / \mathrm{ml})$

Figure 4. siRNA knockdown of Meg3 enhances the cisplatin-resistance of A549 human lung cancer cells. (A) Flow cytometric analysis of apoptosis of A549 (siRNA/control) and siRNA/Meg3-852 cells treated with various concentrations of cisplatin $(0.0,1.0 \mathrm{or} 2.0 \mu \mathrm{g} / \mathrm{ml})$. (B) Flow cytometric analysis of the cell cycle distribution of A549 and siRNA/Meg3-852 cells treated with various concentrations of cisplatin $(0.0,1.0 \mathrm{or} 2.0 \mu \mathrm{g} / \mathrm{ml})$. The results represent the average of three independent experiments (mean \pm standard error of the mean). " $\mathrm{P}<0.05$ vs. siRNA/control cells. siRNA, small interfering RNA; Meg3, maternally expressed gene 3 .

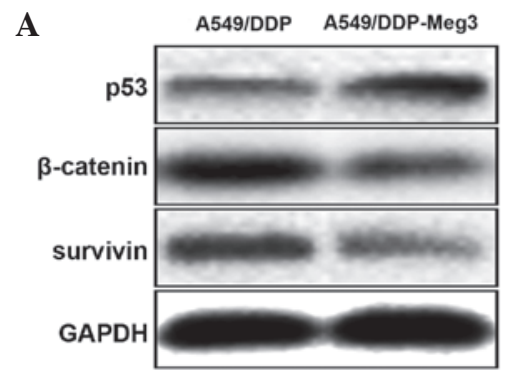

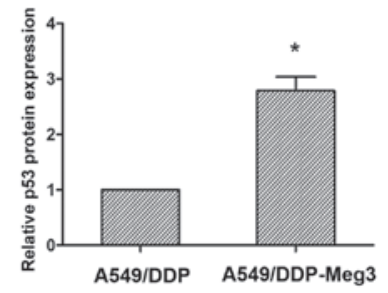

B
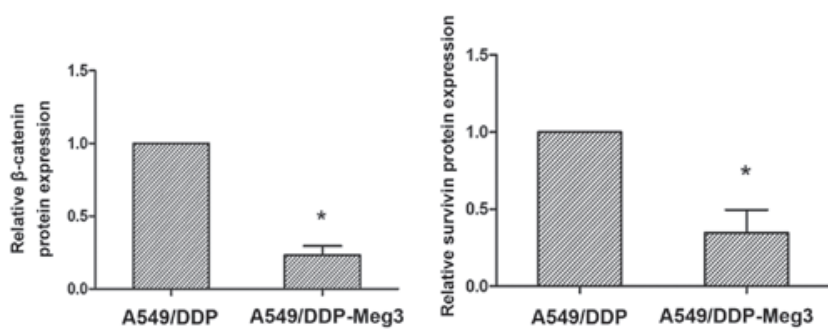

$\mathrm{p}=0.0068$

$n=27$

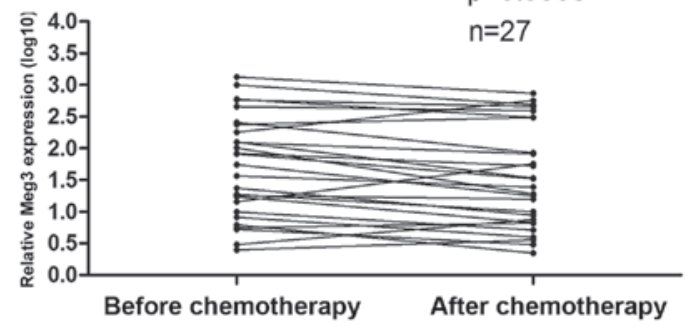

Figure 5. Meg3 regulates p53, $\beta$-catenin and survivin expression, and cisplatin treatment decreases the expression levels of Meg3. (A) Protein expression levels of p53, $\beta$-catenin and survivin in A549/DDP (control) or A549/DDP-Meg3 (Meg3 overexpressing) human lung cancer cells were analyzed by western blotting. GAPDH was used as a control. Average values of integrated optical density were assessed by analyzing five times per experiment and recorded in the histograms. ${ }^{*} \mathrm{P}<0.05$. (B) Expression levels of Meg3 were detected in blood samples ( $\mathrm{n}=27)$ from patients with advanced lung cancer prior to and following treatment with cisplatin-based chemotherapy by reverse transcription-quantitative polymerase chain reaction. GAPDH was used as a reference. Meg3, maternally expressed gene 3 . 
line, as compared with in the A549 parental cell line $(\mathrm{P}<0.05$; Fig. 1B).

Treatment with cisplatin reduces expression of Meg3. The results above demonstrated that the expression levels of Meg3 were upregulated in A549 cells. The present study aimed to determine the expression levels of Meg3 in response to various concentrations of cisplatin in A549 cells. The cells were treated with various concentrations of cisplatin $(0.0,0.5$, $1.0,1.5,2.0$ and $2.5 \mu \mathrm{g} / \mathrm{ml}$ ) for $24 \mathrm{~h}$. The relative expression levels of Meg3 were decreased in response to treatment with increasing concentrations of cisplatin (Fig. 1C). These results suggested that a reduction in Meg3 expression levels in the A549 lung cancer cell line was induced in response to cisplatin treatment.

Meg3 upregulation reverses the cisplatin resistance of A549/DPP cells. To further evaluate the effects of Meg3 on the development of cisplatin resistance in lung cancer cells, a lentivirus overexpressing Meg3 was stably transfected into the A549/DDP cells (A549/DDP-Meg3). The transfection efficiency was validated by RT-qPCR. The expression levels of Meg3 in the A549/DDP-Meg3 cells were significantly upregulated, compared with those of the A549/DDP control cells $(\mathrm{P}<0.05$; Fig. 1D). Furthermore, overexpression of Meg3 led to a reduction in the $\mathrm{IC}_{50}$ of cisplatin in A549/DDP cells by 9.79-fold ( $\mathrm{P}<0.05$; Fig. 1E).

In the A549/DDP-Meg3 cells treated with cisplatin (0.0, 1.0 and $1.5 \mu \mathrm{g} / \mathrm{ml}$ ), upregulation of $\mathrm{Meg} 3$ resulted in an increased rate of cisplatin-induced apoptosis ( $\mathrm{P}<0.05$; Fig. 2A). Furthermore, the percentage of A549/DDP-Meg3 cells in the subG $G_{0} / G_{1}$ and $G_{1}$ phases, particularly at checkpoint $\operatorname{subG}_{0} /{ }_{1}$, of the cell cycle gradually increased with the increasing doses of cisplatin $(\mathrm{P}<0.05$; Fig. 2B). These results suggested that upregulation of Meg3 may reverse the cisplatin resistance of A549/DDP cells by inducing apoptosis and cell cycle arrest.

siRNA knockdown of Meg3 reduces the rate of cisplatin-induced apoptosis and alters cell cycle distribution in A549 cells. A549 cells were transfected with siRNA/control, siRNA/Meg3-243, siRNA/Meg3-405 or siRNA/Meg3-852, in order to analyze the role of Meg3 in determining the sensitivity of lung cancer cells to cisplatin. At $48 \mathrm{~h}$ post-transfection, the expression levels of Meg3 were significantly decreased by $75.5 \%$ in the A549-siRNA/Meg3-852 cells compared with the siRNA/control-transfected cells $(\mathrm{P}<0.01$; Fig. 3A). The Meg3 inhibition efficiency was higher with this siRNA than that of siRNA/Meg3-243 (30.1\%) and siRNA/Meg3-405 (35.3\%). Based on these results, siRNA/Meg3-852 was used to suppress the expression of Meg3 in A549 cells (Fig. 3B). The effects of Meg3 expression on the $\mathrm{IC}_{50}$ of cisplatin in A549 cells were subsequently investigated. An MTT assay demonstrated that siRNA/Meg3-852 increased the $\mathrm{IC}_{50}$ of cisplatin in A549 cells $(\mathrm{P}<0.05$; Fig. 3C). Furthermore, A549-siRNA/Meg3-852 cells were treated with various concentrations of cisplatin $(0.0,1.0$ and $2.0 \mu \mathrm{g} / \mathrm{ml})$. An apoptosis assay demonstrated that knockdown of Meg3 by RNA interference reduced the rate of cisplatin-induced apoptosis in lung cancer cells $(\mathrm{P}<0.05$; Fig. $4 \mathrm{~A})$. In addition, the percentage of siRNA/Meg3-transfected A549 cells in
subG $G_{0} / G_{1}$ and $G_{1}$ phases, particularly in checkpoint $G_{1}$, of the cell cycle increased with increasing doses of cisplatin $(\mathrm{P}<0.05$; Fig. 4B).

Upregulation of Meg3 in A549/DDP cells decreases cisplatin resistance through inhibition of $W N T / \beta$-catenin signaling. A previous study reported that Meg3 was able to regulate the specificity of p53 transcriptional activation (17). The tumor suppressor gene p53 has a central role in tumor suppression, and mediates the functions of numerous tumor suppressors, in particular those associated with apoptosis and drug resistance of cancer cells (18). Furthermore, it has been demonstrated that the overexpression of wild-type p53, by transfection or DNA damage, downregulates the expression of $\beta$-catenin in human cells (19). The inhibitory effects of p53 on $\beta$-catenin have been revealed to be mediated by the ubiquitin-proteasome system and require activation of glycogen synthase kinase-3 $\beta$ (GSK-3 $\beta$ ) (19). WNTs regulate embryonic development and determine cell fate, and dysregulation of $\mathrm{WNT} / \beta$-catenin signaling may induce the development and/or progression of various human diseases, including cancer (20). Therefore, analyzing the effects of potential therapies on the WNT/ $\beta$-catenin signaling pathway may represent a novel method for the screening of putative anti-cancer drugs. Gao et al (21) identified potential drugs that were able to inhibit $\mathrm{WNT} / \beta$-catenin signaling, including cisplatin, using a drug screening platform based on $\beta$-catenin/transcription factor-mediated transcriptional activity. In addition, cisplatin was shown to activate WNT/ $\beta$-catenin signaling in A549/DDP cells, and inactivate it in A549 cells (21). Based on these prior studies, the present study hypothesized that Meg3 may regulate the cisplatin resistance of cancer cells via the inhibition of $\beta$-catenin/survivin by activated p53. To examine this hypothesis, the protein expression levels of $\mathrm{p} 53, \beta$-catenin and survivin were determined in A549/DDP-Meg3 and A549/DDP cells by western blot analysis. Upregulation of $\mathrm{p} 53$ and downregulation of $\beta$-catenin/survivin was observed in the A549/DDP-Meg3 cells, and conversely the expression levels of these proteins were reversed in the A549/DDP cells (Fig. 5A). These findings suggested that upregulation of Meg3 in A549/DDP cells may decrease cisplatin resistance via inhibition of $\mathrm{WNT} / \beta$-catenin signaling.

Meg3 expression levels are downregulated in blood samples from patients with advanced lung cancer following cisplatin-based chemotherapy. In a clinical setting, cisplatin is frequently used to treat patients with advanced lung cancer. In the present study, blood samples $(n=27)$ were collected from patients with advanced lung cancer in order to determine the effects of cisplatin treatment on Meg3 expression. RT-qPCR analysis demonstrated that the expression levels of Meg3 were decreased in patients with advanced lung cancer following treatment with cisplatin-based chemotherapy, compared with those prior to cisplatin-based chemotherapy $(\mathrm{P}=0.0068$; Fig. 5B). These results suggested that Meg3 may have a significant role as a therapeutic target in lung cancer.

\section{Discussion}

Lung cancer is one of the most common causes of cancer-associated mortality worldwide. The postoperative resistance of 
patients to chemotherapy drugs, including cisplatin and TKI, is a significant factor in determining the prognosis of patients with lung cancer. Therefore, exploration into the mechanisms underlying cisplatin resistance are crucial for improving treatment outcomes. IncRNAs are a class of non-coding RNAs, which have been implicated in the development of various cancers. Emerging literature has demonstrated that numerous IncRNAs are associated with the biological processes underlying various types of cancer (22-24). The lncRNA SChLAP1 has been shown to contribute to the development of lethal types of prostate cancer, at least in part by antagonizing the tumor-suppressive functions of the switch/sucrose nonfermentable complex (25). In hepatocellular cancer, the lncRNA low expression in tumor is suppressed by histone deacetylase 3 , and may contribute to hypoxia-mediated metastasis (26). The present study investigated the association of the lncRNA Meg3 with the cell cycle, apoptosis and cisplatin resistance of human lung cancer cell lines.

It remains a major therapeutic challenge amongst numerous patients with cancer who are treated with anti-cancer drugs, that they exhibit significant chemoresistance, including intrinsic and acquired resistance (27). The study of tumor chemotherapy drug resistance has continued to grow; however, the potential mechanisms underlying the resistance to chemotherapeutic agents remain unclear. Recent studies have demonstrated that substantial epigenetic alterations may contribute to facilitating successful chemotherapeutic treatment in drug-resistant cancer cells $(28,29)$. The results of the present study demonstrated that the expression levels of Meg3 were markedly lower in the cisplatin-resistant cell line A549/DDP, as compared with those in the A549 parental cell line. In addition, reduced expression levels of Meg3 were detected in the A549 lung cancer cell line in response to treatment with cisplatin. Furthermore, upregulation of Meg3 resulted in an increased rate of cisplatin-induced apoptosis of A549/DDP-Meg3 cells; whereas, knockdown of Meg3 expression by RNA interference reduced the cisplatin-induced apoptosis of lung cancer cells.

The percentage of A549/DDP-Meg3 cells in $\mathrm{subG}_{0} / \mathrm{G}_{1}$ and $\mathrm{G}_{1}$ phase, particularly at checkpoint $\operatorname{subG}_{0} /{ }_{1}$, of the cell cycle gradually increased with increasing doses of cisplatin, whereas the percentage of siRNA/Meg3 transfected A549 cells in subG ${ }_{0} / G_{1}$ and $G_{1}$ phase, particularly at checkpoint $\mathrm{G}_{1}$, of the cell cycle gradually increased with increasing doses of cisplatin. Braconi et al (30) previously demonstrated that Meg3 inhibited the percentage of hepatocellular cancer cells in subG $_{0} /{ }_{1}$ phase of the cell cycle, without significantly modulating the percentage of cells at checkpoint $\mathrm{G}_{0} /{ }_{1}$ or $\mathrm{G}_{2} / \mathrm{M}$. These results were concordant with the findings of the present study. The tumor suppressor gene p53 contributes to the apoptosis, cell cycle and drug resistance of cancer cells. Meg3 has previously been suggested to regulate the specificity of p53 transcriptional activation (17). Butz and Patoc (31) reported that Meg3 activates p21CIP1 through p53, and its checkpoint is $\mathrm{G}_{1} / \mathrm{S}$ in the pituitary gland. However, Zhou et al (17) reported that Meg3 does not stimulate p21CIP1 expression. These contradictory results suggest that changes in apoptosis, cell cycle distribution and drug resistance of cancer cells should not be attributed to alterations of just one, or a small quantity of genes.
Cisplatin resistance is associated with altered cellular signaling, including the phosphoinositide-3-kinase-Akt, p53, and $\mathrm{WNT} / \beta$-catenin signaling pathways (32-34). $\beta$-catenin is a key component of the WNT signaling pathway and is subsequently phosphorylated by GSK-3 $\beta$, which targets $\beta$-catenin for ubiquitination and proteolytic degradation. Activation of $\mathrm{WNT} / \beta$-catenin signaling inhibits formation of the multi-protein complex, and phosphorylation of $\beta$-catenin by GSK-3 $\beta$. A previous study demonstrated that high levels of survivin inhibited the induction of apoptosis by cisplatin and resulted in drug resistance in A549/DDP cells; however, a lack of survivin enhanced cisplatin-mediated apoptosis in A549 cells (35). Based on the results of previous studies, the present study hypothesized that Meg3 may regulate apoptosis, cell cycle distribution and drug resistance of cancer cells via the inhibition of $\beta$-catenin/survivin by activated $\mathrm{p} 53$. The present study aimed to elucidate the mechanism underlying the effects of Meg3 on apoptosis, cell cycle and drug resistance within the limits of NSCLC. However, the biological and pathological functions of Meg3 in general remain to be determined.

In conclusion, to the best of our knowledge, the present study was the first to demonstrate that the expression of Meg3 was decreased in the A549/DDP cisplatin-resistant cell line, as compared with A549 cells. The expression levels of Meg3 were also shown to be downregulated in blood samples taken from patients with advanced lung cancer following treatment with cisplatin-based chemotherapy. The present study also demonstrated that downregulation of Meg3 enhanced the cisplatin resistance of lung cancer cells through activation of the $\mathrm{WNT} / \beta$-catenin signaling pathway, thus indicating that Meg3 may have a significant role as a therapeutic target in lung cancer.

\section{Acknowledgements}

The authors of the present study would like to thank Dr Junwei Tang at The First Affiliated Hopsital of Nanjing Medical University (Nanjing, China) for language revision.

\section{References}

1. Jemal A, Siegel R, Xu J and Ward E: Cancer statistics, 2010. CA Cancer J Clin 60: 277-300, 2010

2. Sánchez de Cos J, Sojo González MA, Montero MV, et al: Non-small cell lung cancer and silent brain metastasis. Survival and prognostic factors. Lung Cancer 63: 140-145, 2009.

3. Chen CL, Ip SM, Cheng D, Wong LC and Ngan HY: Loss of imprinting of the IGF-II and H19 genes in epithelial ovarian cancer. Clin Cancer Res 6: 474-479, 2000.

4. Yang F, Zhang L, Huo XS, et al: Long noncoding RNA high expression in hepatocellular carcinoma facilitates tumor growth through enhancer of zeste homolog 2 in humans. Hepatology 54: 1679-1689, 2011.

5. Lai MC, Yang Z, Zhou L, et al: Long non-coding RNA MALAT-1 overexpression predicts tumor recurrence of hepatocellular carcinoma after liver transplantation. Med Oncol 29: 1810-1816, 2012.

6. Tsang WP and Kwok TT: Riboregulator H19 induction of MDR1-associated drug resistance in human hepatocellular carcinoma cells. Oncogene 26: 4877-4881, 2007.

7. Fan Y, Shen B, Tan M, et al: Long non-coding RNA UCA1 increases chemoresistance of bladder cancer cells by regulating Wnt signaling. FEBS J 281: 1750-1758, 2014.

8. Jiang M, Huang O, Xie $\mathrm{Z}$, et al: A novel long non-coding RNA-ARA: Adriamycin resistance-associated. Biochem Pharmacol 87: 254-283, 2014. 
9. Gejman R, Batista DL, Zhong Y, et al: Selective loss of MEG3 expression and intergenic differentially methylated region hypermethylation in the MEG3/DLK1 locus in human clinically nonfunctioning pituitary adenomas. J Clin Endocrinol Metab 93: 4119-4125, 2008.

10. Sun M, Xia R, Jin F, et al: Downregulated long noncoding RNA MEG3 is associated with poor prognosis and promotes cell proliferation in gastric cancer. Tumour Biol 35: 1065-1073, 2014.

11. Ying L, Huang Y, Chen H, et al: Downregulated MEG3 activates autophagy and increases cell proliferation in bladder cancer. Mol Biosyst 9: 407-411, 2013.

12. Wang P, Ren Z and Sun P: Overexpression of the long non-coding RNA MEG3 impairs in vitro glioma cell proliferation. J Cell Biochem 113: 1868-1874, 2012

13. Qin R, Chen Z, Ding Y, Hao J, Hu J and Guo F: Long non-coding RNA MEG3 inhibits the proliferation of cervical carcinoma cells through the induction of cell cycle arrest and apoptosis. Neoplasma 60: 486-492, 2013

14. Zhao X, Xu Z, Wang Z, et al.: RNA silencing of integrin-linked kinase increases the sensitivity of the A549 lung cancer cell line to cisplatin and promotes its apoptosis. Mol Med Rep 12: 960-966, 2015

15. Stewart DJ: Wnt signaling pathway in non-small cell lung cancer. J Natl Cancer Inst 106: djt356, 2014.

16. Ward JH: NCCN Guidelines and the International Community. J Natl Compr Canc Netw 9: 133-134, 2011.

17. Zhou Y, Zhong Y, Wang Y, et al: Activation of p53 by MEG3 non-coding RNA. J Biol Chem 282: 24731-24742, 2007.

18. Miao W, Liu X, Wang H, et al: p53 upregulated modulator of apoptosis sensitizes drug-resistant U251 glioblastoma stem cells to temozolomide through enhanced apoptosis. Mol Med Rep 11: $4165-4173,2015$.

19. Sadot E, Geiger B, Oren M and Ben-Ze'ev A: Down-regulation of beta-catenin by activated p53. Mol Cell Biol 21: 6768-6781, 2001.

20. Wu MY, Xie X, Xu ZK, et al: PP2A inhibitors suppress migration and growth of PANC-1 pancreatic cancer cells through inhibition on the Wnt/beta-catenin pathway by phosphorylation and degradation of $\beta$-catenin. Oncol Rep 32: 513-522, 2014.

21. Gao Y, Liu Z, Zhang X, et al: Inhibition of cytoplasmic GSK-3 $\beta$ increases cisplatin resistance through activation of Wnt $/ \beta$-catenin signaling in A549/DDP cells. Cancer Lett 336: 231-239, 2013.

22. Ren S, Liu Y, Xu W, et al: Long noncoding RNA MALAT-1 is a new potential therapeutic target for castration resistant prostate cancer. J Urol 190: 2278-2287, 2013.
23. Schmidt LH, Spieker T, Koschmieder S, et al: The long noncoding MALAT-1 RNA indicates a poor prognosis in non-small cell lung cancer and induces migration and tumor growth. $\mathrm{J}$ Thorac Oncol 6: 1984-1992, 2011

24. Xi YN, Xin XY and Ye HM: Effects of HMGA2 on malignant degree, invasion, metastasis, proliferation and cellular morphology of ovarian cancer cells. Asian Pac J Trop Med 7: 289-292, 2014

25. Prensner JR, Iyer MK, Sahu A, et al: The long noncoding RNA SChLAP1 promotes aggressive prostate cancer and antagonizes the SWI/SNF complex. Nat Genet 45: 1392-1398, 2013.

26. Yang F, Huo XS, Yuan SX, et al: Repression of the long noncoding RNA-LET by histone deacetylase 3 contributes to hypoxia-mediated metastasis. Mol Cell 49: 1083-1096, 2013.

27. Chang A: Chemotherapy, chemoresistance and the changing treatment landscape for NSCLC. Lung Cancer 71: 3-10, 2011.

28. Strathdee G: Epigenetic markers and response to chemotherapy in cancer. Dis Markers 23: 43-49, 2007.

29. Liu Z, Sun M, Lu K, et al: The long noncoding RNA HOTAIR contributes to cisplatin resistance of human lung adenocarcinoma cells via downregualtion of p21 (WAF1/CIP1) expression. PloS One 8: e77293, 2013.

30. Braconi C, Kogure T, Valeri N, et al: microRNA-29 can regulate expression of the long non-coding RNA gene MEG3 in hepatocellular cancer. Oncogene 30: 4750-4756, 2011.

31. Butz $\mathrm{H}$ and Patocs A: Pituitary tumorigenesis: role of regulation of Weel kinase by microRNAs. In: Tumors of the Central Nervous System. Hayat M (ed). Vol 10. Springer, Dordrecht, pp141-150, 2013.

32. McCubrey JA, Steelman LS, Abrams SL, et al: Roles of the RAF/MEK/ERK and PI3K/PTEN/AKT pathways in malignant transformation and drug resistance. Adv Enzyme Regul 46: 249-279, 2006

33. Bouwman P and Jonkers J: The effects of deregulated DNA damage signalling on cancer chemotherapy response and resistance. Nat Rev Cancer 12: 587-598, 2012.

34. Cui J, Jiang W, Wang S, Wang L and Xie K: Role of Wnt/ $\beta$-catenin signaling in drug resistance of pancreatic cancer. Curr Pharm Des 18: 2464-2471, 2012.

35. Yang D, Welm A and Bishop JM: Cell division and cell survival in the absence of survivin. Proc Natl Acad Sci USA 101: 15100-15105, 2004. 